MATHEMATICS OF COMPUTATION

Volume 76, Number 258, April 2007, Pages 1025-1038

S 0025-5718(06)01958-2

Article electronically published on December 29, 2006

\title{
ON THE SMALLEST VALUE OF THE MAXIMAL MODULUS OF AN ALGEBRAIC INTEGER
}

\author{
GEORGES RHIN AND QIANG WU
}

\begin{abstract}
The house of an algebraic integer of degree $d$ is the largest modulus of its conjugates. For $d \leq 28$, we compute the smallest house $>1$ of degree $d$, say $\mathrm{m}(d)$. As a consequence we improve Matveev's theorem on the lower bound of $\mathrm{m}(d)$. We show that, in this range, the conjecture of SchinzelZassenhaus is satisfied. The minimal polynomial of any algebraic integer $\boldsymbol{\alpha}$ whose house is equal to $\mathrm{m}(d)$ is a factor of a bi-, tri- or quadrinomial. The computations use a family of explicit auxiliary functions. These functions depend on generalizations of the integer transfinite diameter of some compact sets in $\mathbb{C}$. They give better bounds than the classical ones for the coefficients of the minimal polynomial of an algebraic integer $\boldsymbol{\alpha}$ whose house is small.
\end{abstract}

\section{INTRODUCTION}

Let $\boldsymbol{\alpha}$ be a nonzero algebraic integer of degree $d$, whose conjugates are $\boldsymbol{\alpha}_{1}=\boldsymbol{\alpha}$, $\boldsymbol{\alpha}_{2}, \ldots, \boldsymbol{\alpha}_{d}$, and let

$$
P=b_{0} X^{d}+b_{1} X^{d-1}+\cdots+b_{d-1} X+b_{d},
$$

with $b_{0}=1$, be its minimal polynomial. We denote, as usual, by

$$
|\boldsymbol{\alpha}|=\max _{1 \leq i \leq d}\left|\boldsymbol{\alpha}_{i}\right|
$$

the house of $\boldsymbol{\alpha}$, and by $\nu$ the number of $\boldsymbol{\alpha}_{i}$ such that $\left|\boldsymbol{\alpha}_{i}\right|>1$. Then $|\boldsymbol{\alpha}| \geq 1$ and Kronecker's theorem asserts that $\mid \boldsymbol{\alpha}=1$ if and only if $\boldsymbol{\alpha}$ is a root of unity. We define $\mathrm{m}(d)$ to be the minimum of the houses of the algebraic integers $\boldsymbol{\alpha}$ of degree $d$ which are not a root of unity.

A classical problem, see P. Borwein $[\mathrm{PB}$, is to study the behaviour of $\mathrm{m}(d)$ when $d$ varies. On the one hand, it is clear that $\mathrm{m}(d) \leq 2^{1 / d}$ since the polynomial $X^{d}-2$ is irreducible of degree $d$. On the other hand, there is a conjecture of A. Schinzel and H. Zassenhaus $\mathrm{SZ}$ which asserts that $\mathrm{m}(d) \geq 1+c_{1} / d$, where $c_{1}$ is a positive constant. Moreover D. Boyd $\left[\mathrm{DB}\right.$ suggests that $c_{1}$ should be equal to $\frac{3}{2} \log \left(\theta_{0}\right)$ where $\theta_{0}=1.3247 \ldots$ is the smallest Pisot number which is the real root of $X^{3}-X-1$. This is based on the fact, pointed out by C.J. Smyth, that for $d=3 k$ the number $\boldsymbol{\alpha}$ with minimal polynomial $X^{3 k}+X^{2 k}-1$ has $|\boldsymbol{\alpha}|=\theta_{0}^{1 /(2 k)}=\theta_{0}^{3 /(2 d)}$,

Received by the editor December 24, 2005 and, in revised form, December 28, 2005.

2000 Mathematics Subject Classification. Primary 11C08, 11R06, 11 Y 40.

Key words and phrases. Algebraic integer, maximal modulus, Schinzel-Zassenhaus conjecture, Mahler measure, Smyth's theorem, Perron numbers, explicit auxiliary functions, integer transfinite diameter.

Qiang Wu was supported in part by the Natural Science Foundation of Chongqing grant CSTC no. 2005BB8024. 
and it is expected that this is equal to $\mathrm{m}(d)$ for this degree. We say that an $\boldsymbol{\alpha}$ which gives $\mathrm{m}(d)$ is extremal.

We define the Mahler measure of $\boldsymbol{\alpha}$ (and of $P$ ) by

$$
\mathrm{M}(\boldsymbol{\alpha})=\left|b_{0}\right| \prod_{i=1}^{d} \max \left(1,\left|\alpha_{i}\right|\right) .
$$

We say that $\boldsymbol{\alpha}$ is reciprocal if $\boldsymbol{\alpha}^{-1}$ is a conjugate of $\boldsymbol{\alpha}$. Smyth SM proved that, if $\alpha \neq 0,1$ is nonreciprocal, then $\mathrm{M}(\boldsymbol{\alpha}) \geq \theta_{0}$. Since $\mathrm{M}(\boldsymbol{\alpha}) \leq|\boldsymbol{\alpha}|^{d}$, in this case we have $|\boldsymbol{\alpha}| \geq 1+\log \left(\theta_{0}\right) / d$.

P. Voutier $[\mathrm{V}]$ proved that, if $\boldsymbol{\alpha}$ is a nonzero algebraic integer of degree $d \geq 3$ which is not a root of unity, then

$$
\mathrm{M}(\alpha) \geq 1+\frac{1}{4}\left(\frac{\log \log d}{\log d}\right)^{3} .
$$

This gives

$$
\mathrm{m}(d) \geq\left(1+\frac{1}{4}\left(\frac{\log \log d}{\log d}\right)^{3}\right)^{1 / d} .
$$

A. Dubickas [D] showed that the constant $1 / 4$ in (1.1) can be replaced by $64 / \pi^{2}-\varepsilon$ if $d>d_{0}(\varepsilon)$.

E. M. Matveev [MAT] proved the following result:

Theorem 1. Let $\boldsymbol{\alpha}$ be an algebraic integer, not a root of unity, and let $d=$ $\operatorname{deg}(\boldsymbol{\alpha}) \geq 2$. Then

$$
\left\lceil\boldsymbol{\alpha} \geq \exp \left(\log (d+0.5) / d^{2}\right) .\right.
$$

Moreover, if $\boldsymbol{\alpha}$ is reciprocal and $d \geq 6$, then

$$
|\boldsymbol{\alpha}| \geq \exp \left(3 \log (d / 2) / d^{2}\right) .
$$

The lower bound for $\mathrm{m}(d)$ given in (1.1) is asymptotically better than the bound given in (1.2), but improves it only for $d \geq 1435$. Hence, for $d$ not too large, the inequality (1.2) is better than (1.1).

For $d \geq 29$, we have $\exp \left(3 \log (d / 2) / d^{2}\right) \leq \theta_{0}{ }^{1 / d}$. So by Smyth's theorem, any nonzero algebraic integer $\boldsymbol{\alpha}$ of degree $d$, not a root of unity, whose house is less than $\theta_{0}{ }^{1 / d}$ is reciprocal. Then we may apply the second part of Matveev's theorem. Hence we deduce that, for $d \geq 29$,

$$
\mathrm{m}(d) \geq \exp \left(3 \log (d / 2) / d^{2}\right) .
$$

In this paper our goal is, on the one hand, to verify the conjecture of Schinzel and Zassenhaus with Boyd's constant up to $d=28$. On the other hand, we use these results to prove an interesting consequence of Matveev's theorem:

Theorem 2. Let $\boldsymbol{\alpha}$ be a nonzero algebraic integer, not a root of unity, and $d=\operatorname{deg}(\boldsymbol{\alpha}) \geq 4$.

Then for $d \leq 12$,

$$
|\boldsymbol{\alpha}| \geq \exp \left(3 \log (d / 3) / d^{2}\right)
$$

and for $d \geq 13$,

$$
\mid \boldsymbol{\alpha} \geq \exp \left(3 \log (d / 2) / d^{2}\right)
$$


This result gives a better lower bound than (1.1) for $d \leq 6380$.

As a consequence of our computations we get the following results:

Proposition. 1. The conjecture of Schinzel and Zassenhaus is true, with $c_{1}=$ $\frac{3}{2} \log \left(\theta_{0}\right)$, for $1 \leq d \leq 28$.

2. For $1 \leq d \leq 28, \mathrm{~m}(d)$ is given by a polynomial which is a factor of a polynomial with at most four monomials.

3. For $3 \leq d \leq 30, \mathrm{~m}(d)$ is stricly less than $2^{1 / d}$.

For degree 31 we have computed the houses of all irreducible polynomials of height 1 . They are all $>2^{1 / 31}$. So we expect the following.

Conjecture. $m(31)=2^{1 / 31}$.

A Perron number is a positive algebraic integer $\boldsymbol{\alpha}$ of degree $d$ such that $\boldsymbol{\alpha}>$ $\max _{2 \leq i \leq d}\left|\boldsymbol{\alpha}_{i}\right|$. For the degrees $d=17$ and $d=23$ the extremal $\boldsymbol{\alpha}$ is a Perron number. Hence they satisfy the conjecture of Lind-Boyd [DB]:

Conjecture (Lind-Boyd). The smallest Perron number of degree $d \geq 2$ has minimal polynomial

$$
\begin{aligned}
& X^{d}-X-1 \text { if } d \not \equiv 3,5 \bmod 6, \\
& \left(X^{d+2}-X^{4}-1\right) /\left(X^{2}-X+1\right) \text { if } d \equiv 3 \bmod 6 \text {, } \\
& \left(X^{d+2}-X^{2}-1\right) /\left(X^{2}-X+1\right) \text { if } d \equiv 5 \bmod 6 \text {. }
\end{aligned}
$$

Boyd [DB] also made the following conjecture:

Conjecture (Boyd). 1. The extremal $\boldsymbol{\alpha}$ is always nonreciprocal.

2. If $d=3 k$, then the extremal $\boldsymbol{\alpha}$ has minimal polynomial $X^{3 k}+X^{2 k}-1$ (or $\left.X^{3 k}-X^{2 k}+1\right)$.

3. The extremal $\boldsymbol{\alpha}$ of degree $d$ has $\nu \sim \frac{2}{3} d$ as $d \rightarrow \infty$.

We give in Table 1 the list of extremal $\boldsymbol{\alpha}$ for $d=1$ to $d=28$. We see that the claims 1 and 2 in Boyd's conjecture are satisfied. Boyd noticed that, up to degree $12, \nu(d)$ is monotone, but this is no longer true for $d>13$. The minimal polynomial of the extremal $\boldsymbol{\alpha}$ for degree 23 is

$$
\begin{gathered}
P_{23}=X^{23}+X^{22}-X^{20}-X^{19}+X^{17}+X^{16}-X^{14}-X^{13} \\
+X^{11}+X^{10}-X^{8}-X^{7}+X^{5}+X^{4}-X^{2}-X-1 .
\end{gathered}
$$

Since this is a Perron number which satisfies the conjecture of Lind-Boyd, it can be written as

Likewise,

$$
P_{23}=\frac{X^{25}-X^{2}-1}{X^{2}-X+1}
$$

$$
P_{19}=X^{19}+X^{18}+X^{15}+X^{14}+X^{11}+X^{10}-X^{8}+X^{6}-X^{4}+X^{2}-1
$$

can be written as

$$
P_{19}=\frac{X^{22}-X^{11}-X+1}{X^{3}-X^{2}+X-1}
$$

Therefore, for any extremal $\boldsymbol{\alpha}$ of degree $d$, we write its minimal polynomial $P_{d}$ as a simple polynomial divided by a product of cyclotomic polynomials. 
We make the following conjecture:

Conjecture. Any extremal $\boldsymbol{\alpha}$ has minimal polynomial which is a factor of a polynomial which has at most 4 monomials.

Proof of Theorem 2: We have only to verify that, for $4 \leq d \leq 28, \mathrm{~m}(d)$ is greater than the right-hand side in (1.4) or (1.5).

Boyd [DB] has computed the smallest houses for $d \leq 12$. The main tool in his computations is to give bounds for $s_{k}$, which is the sum of the $k$-th powers of the roots of $P$, for $1 \leq k \leq 3 d$. If $|\boldsymbol{\alpha}| \leq B$, then $\left|s_{k}\right| \leq d B^{k}$. He uses these bounds together with Newton's formula:

$$
s_{k}+s_{k-1} b_{1}+\ldots+s_{1} b_{k-1}+k b_{k}=0,
$$

which gives by induction bounds for the coefficients $b_{k}$, for $1 \leq k \leq d$, in order to get a large set $\mathcal{F}_{d}$ of polynomials. He computes $s_{k}$ with Newton's formula for $d+1 \leq k \leq 3 d$ (with $b_{k}=0$ ) and, for every $k$, he eliminates the polynomial when $s_{k}$ is not within its bounds. So he gets a smaller set $\mathcal{F}_{3 d}$.

We use this principle, but the computing time grows exponentially with the degree $d$. Therefore, to obtain better bounds for the numbers $s_{k}$ we construct a large family of explicit auxiliary functions. These functions are related to suitable generalizations of the integer transfinite diameter of some compact subsets of the complex plane. This method has been used in FRSE to compute all algebraic integers with small Mahler measure up to degree 40.

Here we prove that we may restrict our search to algebraic integers which are units. This property is used to reduce the numbers of polynomials to examine. A priori we cannot assume that this is true for the smallest Perron numbers; therefore the study of smallest Perron numbers will be devoted to a forthcoming paper [WU2].

This paper is organized as follows. In Section 2 we prove that, up to degree 30, for any degree $d$ there exists an algebraic integer such that $1<|\boldsymbol{\alpha}|<2^{1 / d}$. This proves that $\left|b_{d}\right|=1$. In Section 3 we show how to use explicit auxiliary functions to give bounds for $s_{k}$. We also explain how to construct such auxiliary functions. In Section 4 we give some refinements of the previous method. We give relations between $s_{k}$ and $s_{2 k}$. Moreover, for $d=26$ and $d=28$ we show that the triples $\left(b_{d-2}, b_{d-1}, b_{d}\right)$ belong to a rather "small" set. Section 5 is devoted to the final computations. The search for the degree 28 took 6800 hours on a $2.8 \mathrm{Ghz}$ PC.

The first author is gratefully indebted to the South West University of China in Chongqing where a great part of this work was done.

\section{The NORM OF $\boldsymbol{\alpha}$ With SMALlest house FOR $d \leq 30$}

To prove the previous assertion for $3 \leq d \leq 30$, it suffices to prove it for $d=4$ and when $d$ is an odd prime $\leq 29$ because, if $\operatorname{deg}(\boldsymbol{\alpha})=d$ and $|\boldsymbol{\alpha}|<2^{1 / d}$, then $\left|\boldsymbol{\alpha}^{1 / k}\right|<2^{1 / d k}$. For $4 \leq d \leq 23$ the results can be found in Table 1 . When $P_{d}$ is a primitive polynomial, it is written, for $d>3$, as a quotient with numerator a tri- or a quadrinomial. The denominator is a product of at most two cyclotomic polynomials: $\Phi_{1}=X-1, \Phi_{4}=X^{2}+1$ and $\Phi_{6}=X^{2}-X+1$. When $P_{d}$ is not primitive, it is written as $P_{e}\left(X^{k}\right)$ with $d=e k$. For $d=29$ the minimal polynomial of the number $\boldsymbol{\alpha}$ which gives the smallest house $(=1.02338300 \ldots)$ that we have 
TABLe 1. List of extremal $\boldsymbol{\alpha}$ of degree $d$ and minimal polynomial $P_{d}(X)$. In the last column $\nu$ is the number of roots of $P_{d}$ outside the unit disc.

\begin{tabular}{|c|c|c|c|}
\hline$d$ & polynomial $P_{d}(X)$ & $\mathrm{m}(d)$ & $\nu$ \\
\hline 1 & $X-2$ & 2 & 1 \\
\hline 2 & $X^{2}-2$ & $2^{1 / 2}=1.41421356$ & 2 \\
\hline 3 & $X^{3}+X^{2}-1$ & $\theta_{0}^{1 / 2}=1.15096392 \ldots$ & 2 \\
\hline 4 & $\left(X^{5}-X^{3}+X-1\right) / \Phi_{1}$ & 1. $18375181 \ldots$ & 2 \\
\hline 5 & $\left(X^{7}-X^{4}+X+1\right) / \Phi_{4}$ & $1.12164517 \ldots$ & 4 \\
\hline 6 & $P_{3}\left(X^{2}\right)$ & $\theta_{0}^{1 / 4}=1.07282986$ & 4 \\
\hline 7 & $\left(X^{10}-X^{5}-X^{3}+1\right) /\left(\Phi_{1} \Phi_{4}\right)$ & $1.09284559 \ldots$ & 4 \\
\hline 8 & $\left(X^{11}-X^{6}+X-1\right) /\left(\Phi_{1} \Phi_{4}\right)$ & $1.07562047 \ldots$ & 6 \\
\hline 9 & $P_{3}\left(X^{3}\right)$ & $\theta_{0}^{1 / 6}=1.04798219$ & 6 \\
\hline 10 & $P_{5}\left(X^{2}\right)$ & $1.05907751 \ldots$ & 8 \\
\hline 11 & $\left(X^{14}-X^{7}-X+1\right) /\left(\Phi_{1} \Phi_{4}\right)$ & $1.05712485 \ldots$ & 8 \\
\hline 12 & $P_{3}\left(X^{4}\right)$ & $\theta_{0}^{1 / 8}=1.03577500 \ldots$ & 8 \\
\hline 13 & $\left(X^{15}-X^{8}+X+1\right) / \Phi_{4}$ & $1.05372001 \ldots$ & 10 \\
\hline 14 & $P_{7}\left(X^{2}\right)$ & $1.04539255 \ldots$ & 8 \\
\hline 15 & $P_{3}\left(X^{5}\right)$ & $\theta_{0}^{1 / 10}=1.02851905 \ldots$ & 10 \\
\hline 16 & $P_{8}\left(X^{2}\right)$ & $1.03712124 \ldots$ & 12 \\
\hline 17 & $\left(X^{19}-X^{2}-1\right) / \Phi_{6}$ & 1. $03930211 \ldots$ & 11 \\
\hline 18 & $P_{3}\left(X^{6}\right)$ & $\theta_{0}^{1 / 12}=1.02371001 \ldots$ & 12 \\
\hline 19 & $\left(X^{22}-X^{11}-X+1\right) /\left(\Phi_{1} \Phi_{4}\right)$ & 1. $03641032 \ldots$ & 14 \\
\hline 20 & $P_{5}\left(X^{4}\right)$ & $1.02911491 \ldots$ & 16 \\
\hline 21 & $P_{3}\left(X^{7}\right)$ & $\theta_{0}^{1 / 14}=1.02028875 \ldots$ & 14 \\
\hline 22 & $P_{11}\left(X^{2}\right)$ & $1.02816577 \ldots$ & 16 \\
\hline 23 & $\left(X^{25}-X^{2}-1\right) / \Phi_{6}$ & $1.02932014 \ldots$ & 15 \\
\hline 24 & $P_{3}\left(X^{8}\right)$ & $\theta_{0}^{1 / 16}=1.01773032 \ldots$ & 16 \\
\hline 25 & $P_{5}\left(X^{5}\right)$ & 1. $02322489 \ldots$ & 20 \\
\hline 26 & $P_{13}\left(X^{2}\right)$ & 1. $02650865 \ldots$ & 20 \\
\hline 27 & $P_{3}\left(X^{9}\right)$ & $\theta_{0}^{1 / 18}=1.01574486 \ldots$ & 18 \\
\hline 28 & $P_{7}\left(X^{4}\right)$ & $1.02244440 \ldots$ & 16 \\
\hline
\end{tabular}


found for this degree is

$$
\begin{gathered}
P=X^{29}+X^{28}-X^{26}-X^{25}+X^{23}+X^{22}-X^{20}-X^{19}+X^{17} \\
+X^{16}-X^{14}-X^{13}+X^{11}+X^{10}-X^{8}-X^{7}+X^{5}+X^{4}-X^{2}-X-1,
\end{gathered}
$$

which may be written as

$$
P=\frac{X^{31}-X^{2}-1}{X^{2}-X+1} .
$$

In order to prove this assertion and to get good bounds $B$ for our further computations, we seek all houses of irreducible polynomials of height 1 whose house is less than $2^{1 / d}$ from degree 13 to degree 31 . Then, in the sequel, the bound $B$ will be taken, for any $d$, equal to the smallest house we have found during this computation. We use this bound to compute the auxiliary functions of Sections 3 and 4 .

\section{THE BOUNDS FOR $s_{k}$}

a. We consider an explicit auxiliary function $f$ of the following type:

$$
f(z)=-\operatorname{Re}(z)-\sum_{1 \leq j \leq J} e_{j} \log \left|Q_{j}(z)\right|,
$$

where $z$ is a complex number, the $e_{j}$ are positive real numbers and the integer polynomials $Q_{j}$ belong to a fixed set $S$ that will be defined later. The numbers $e_{j}$ are always chosen to get the best auxiliary function. We denote by $m$ the minimum of $f(z)$ for $|z| \leq B$. Since the function $f$ is harmonic in this disc outside the union of small discs around the roots of the polynomials $Q_{j}$, this minimum is taken over $|z|=B$.

We now assume that the polynomial $P$ does not divide any polynomial $Q_{j}\left( \pm X^{k}\right)$ for $1 \leq k \leq 3 d$. Then

$$
\sum_{1 \leq i \leq d} f\left(\boldsymbol{\alpha}_{i}\right) \geq m d
$$

and

$$
-s_{1} \geq d m+\sum_{1 \leq j \leq J} e_{j} \log \left|\prod_{1 \leq i \leq d} Q_{j}\left(\boldsymbol{\alpha}_{i}\right)\right| .
$$

$\prod_{1 \leq i \leq d} Q_{j}\left(\boldsymbol{\alpha}_{i}\right)$ is equal to the resultant of $P$ and $Q_{j}$. Since $P$ does not divide $Q_{j}$, this is a nonzero integer. Therefore

$$
s_{1} \leq-d m .
$$

By symmetry, the same inequality is valid for $-s_{1}$. If we replace $B$ by $B^{k}$ and the numbers $\boldsymbol{\alpha}_{i}$ by the numbers $\pm \boldsymbol{\alpha}_{i}{ }^{k}$, we get upper bounds for $\pm s_{k}$.

Remark. In his proof of Theorem 1, Matveev used an auxiliary function of this type with the four polynomials: $X, X-1, X-2$ and $X^{2}-X-1$.

b. Relations between explicit auxiliary functions and the integer transfinite diameter.

If, inside the auxiliary function (3.1), we replace the real numbers $e_{j}$ by rational numbers we may write

$$
f(z)=-\operatorname{Re}(z)-\frac{t}{h} \log |H(z)|,
$$


where $H$ is in $\mathbb{Z}[X]$ of degree $h$ and $t$ is a positive real number. We want to get a function $f$ whose minimum $m$ in $|z| \leq B$ is as large as possible. That is to say, we seek a polynomial $H \in \mathbb{Z}[X]$ such that

$$
\sup _{|z| \leq B}|H(z)|^{t / h} e^{\operatorname{Re}(z)} \leq e^{-m} .
$$

Now, if we suppose that $t$ is fixed, say $t=1$, it is clear that we need an effective upper bound for the quantity

$$
t_{\mathbb{Z}, \boldsymbol{\varphi}}(|z| \leq B)=\liminf _{\substack{h \geq 1 \\ h \rightarrow \infty}} \inf _{\substack{H \in \mathbb{Z}[X] \\ \operatorname{deg} H=h}} \sup _{|z| \leq B}|H(z)|^{t / h} \boldsymbol{\varphi}(z),
$$

where we use the weight $\varphi(z)=e^{\operatorname{Re}(z)}$. To get an upper bound for $t_{\mathbb{Z}, \boldsymbol{\varphi}}(|z| \leq B)$, it suffices to get an explicit polynomial $H \in \mathbb{Z}[X]$ and then to use the sequence of the successive powers of $H$.

This is a generalization of the integer transfinite diameter. For any $h \geq 1$ we say that a polynomial $H$ (not always unique) is an Integer Chebyshev Polynomial if the quantity $\sup _{|z| \leq B}|H(z)|^{t / h} \boldsymbol{\varphi}(z)$ is a minimum. With Wu's algorithm [WU1, we compute polynomials $H$ of degree less than 30 or 40 and take their irreducible factors as polynomials $Q_{j}$. We start with the polynomial $X-1$, get the best $e_{1}$ and take $t=e_{1}$. After computing $J$ polynomials, we optimize the numbers $e_{j}$ as explained in the next subsection. This gives us a new number $t$, and we continue by induction to get $J+1$ polynomials. The list of polynomials $Q_{j}$ of the set $S$ is given in Table 2.

c. Optimization of the numbers $e_{j}$.

We give a brief scheme of the semi-infinite linear programming method introduced into number theory by C. J. Smyth. More details can be found in FRSE].

To optimize the numbers $e_{j}$, we first put the coefficient of $\operatorname{Re}(z)$ equal to $e_{0}=1$. We take a set $X_{1}$ of "well distributed" points of modulus equal to $B$. By linear programming, we get the maximum $\mu$ of the minimum of a finite set of linear forms whose coefficients are $-\operatorname{Re}\left(z_{i}\right)$ and $-\log \left|Q_{j}\left(z_{i}\right)\right|$ for $1 \leq j \leq J$ for any $z_{i}$ in $X_{1}$. This gives an auxiliary function $f$ which has a minimum $m>\mu$. We add to $X_{1}$ a selection of the points of $|z|=B$ where $f$ has a local minimum. With this new set $X_{2}$ we get another value for $m$ and $\mu$. We stop the process when the integer parts of $m$ and $\mu$ coincide.

d. A refinement for the bounds of $s_{k}$.

When $B^{k}$ becomes too large (say for $k \sim 2 d$ ) the bounds given by the auxiliary functions are not as good as for small $k$. We give now better bounds. For this we need the lemma:

Lemma 1. Let $d \geq 2$ be an integer, $b \geq 1$ a real number and $\boldsymbol{\alpha}_{1}, \ldots, \boldsymbol{\alpha}_{d} d$ positive real numbers satisfying the following properties:

$\boldsymbol{\alpha}_{i} \leq b$ for $1 \leq i \leq d$ and $\prod_{1 \leq i \leq d} \boldsymbol{\alpha}_{i}=1$.

Then we have

$$
\sum_{1 \leq i \leq d} \boldsymbol{\alpha}_{i} \leq(d-1) b+\frac{1}{b^{d-1}}
$$

Proof. We may assume that

$$
\frac{1}{b^{d-1}} \leq \boldsymbol{\alpha}_{1} \leq \ldots \leq \boldsymbol{\alpha}_{d} \leq b,
$$


TABLE 2. List of polynomials $Q_{j}$ of the set $S$ which are used in the auxiliary functions of Section $3, d_{j}=\operatorname{deg} Q_{j}$, and the coefficients of $Q_{j}$ are written from degree $d_{j}$ to 0 .

\begin{tabular}{rrrrrrrrr}
$Q_{j}$ & $d_{j}$ & & \multicolumn{2}{l}{ Coefficients of $Q_{j}$} & \\
$Q_{1}$ & 1 & 1 & -1 & & & & \\
$Q_{2}$ & 1 & 1 & -2 & & & & \\
$Q_{3}$ & 1 & 1 & -3 & & & & \\
$Q_{4}$ & 1 & 1 & -4 & & & & \\
$Q_{5}$ & 1 & 1 & -5 & & & & \\
$Q_{6}$ & 1 & 1 & -6 & & & & \\
$Q_{7}$ & 1 & 1 & -7 & & & & \\
$Q_{8}$ & 2 & 1 & 0 & 1 & & & & \\
$Q_{9}$ & 2 & 1 & -1 & 1 & & & \\
$Q_{10}$ & 2 & 1 & -2 & 2 & & & \\
$Q_{11}$ & 2 & 1 & -3 & 3 & & & \\
$Q_{12}$ & 2 & 1 & -4 & 5 & & & \\
$Q_{13}$ & 2 & 1 & -5 & 7 & & & \\
$Q_{14}$ & 2 & 2 & -4 & 3 & & & \\
$Q_{15}$ & 3 & 1 & -1 & 0 & 1 & & \\
$Q_{16}$ & 4 & 1 & 0 & -1 & 0 & 1 & & \\
$Q_{17}$ & 4 & 1 & -1 & 1 & -1 & 1 & & \\
$Q_{18}$ & 4 & 1 & -2 & 3 & -3 & 2 & & \\
$Q_{19}$ & 4 & 1 & -3 & 5 & -5 & 3 & & \\
$Q_{20}$ & 4 & 1 & -4 & 8 & -9 & 5 & & \\
$Q_{21}$ & 6 & 1 & 0 & 0 & 1 & 0 & 0 & 1
\end{tabular}

whence

$$
1 \leq \boldsymbol{\alpha}_{d} \leq b
$$

If $\boldsymbol{\alpha}_{1}=1$, then $\boldsymbol{\alpha}_{1}=\ldots=\boldsymbol{\alpha}_{d}=1$ and

$$
\sum_{1 \leq i \leq d} \boldsymbol{\alpha}_{i}=d \leq(d-1) b+\frac{1}{b^{d-1}}
$$

since the right-hand side in (3.5) is an increasing function of $b$ on $[1, \infty)$. If $\boldsymbol{\alpha}_{1}=$ $\frac{1}{b^{d-1}}$, then

$$
\boldsymbol{\alpha}_{2}=\ldots=\boldsymbol{\alpha}_{d}=b
$$

and we get (3.4). If $\frac{1}{b^{d-1}}<\boldsymbol{\alpha}_{1}<1$, then there exists an integer $k \geq 1$ such that

$$
\frac{1}{b^{d-1}}<\boldsymbol{\alpha}_{1} \leq \ldots \leq \boldsymbol{\alpha}_{k}<\boldsymbol{\alpha}_{k+1}=\ldots=\boldsymbol{\alpha}_{d}=b .
$$

Let $\gamma$ be a real number such that $\boldsymbol{\alpha}_{1} \gamma \boldsymbol{\alpha}_{k}=1$. Then we have $\gamma \boldsymbol{\alpha}_{k}^{2} \geq 1$. Since

$$
\boldsymbol{\alpha}_{1}+\boldsymbol{\alpha}_{k}=\frac{1}{\gamma \boldsymbol{\alpha}_{k}}+\boldsymbol{\alpha}_{k}
$$

is an increasing function of $\boldsymbol{\alpha}_{k}$, we get (3.4) if we increase successively $\boldsymbol{\alpha}_{k}, \ldots, \boldsymbol{\alpha}_{2}$ until they reach $b$. 
TABLE 3 .

\begin{tabular}{|c|c|c|c|c|c|c|c|c|c|c|c|}
\hline$k$ & 1 & 2 & 3 & 4 & 8 & 16 & 28 & 48 & 60 & 76 & 84 \\
\hline$s_{k}$ & 0 & 0 & 0 & -4 & 4 & -12 & 24 & 36 & -44 & 72 & 24 \\
\hline $\max$ & 5 & 6 & 7 & 8 & 13 & 23 & 41 & 74 & 102 & 145 & 174 \\
\hline MAX & 28 & 29 & 29 & 30 & 33 & 39 & 52 & 81 & 106 & 151 & 180 \\
\hline
\end{tabular}

Since $b_{d}= \pm 1$, we may apply Lemma 1 to the set $\left|\boldsymbol{\alpha}_{i}\right|, 1 \leq i \leq d$, of the moduli of the roots of $P$. Thus we get

$$
\left|s_{k}\right| \leq(d-1) B^{k}+\frac{1}{B^{k(d-1)}} .
$$

This is better than the previous bounds that we have computed with the explicit auxiliary functions.

e. A numerical example for degree 28 .

We give, in Table 3 , the bounds that we obtain for $\left|s_{k}\right|(\max )$ for some values of $k$ and the corresponding classical bounds (MAX). We give also the values of $s_{k}$ for the polynomial $P_{28}$.

\section{IMPROVEMENTS OF THE METHOD}

a. Relations between the bounds for $s_{k}$ and the bounds for $s_{2 k}$.

The classical inequality relating $s_{k}$ and $s_{2 k}$ is the following [DB]:

$$
s_{2 k} \geq \frac{2 s_{k}^{2}}{d}-d B^{2 k}
$$

Here we exploit the relations between $s_{k}$ and $s_{2 k}$ that will be given by explicit auxiliary functions of the following type:

$$
f(z)=\operatorname{Re}\left(z^{2}\right)-e_{0} \operatorname{Re}(z)-\sum_{1 \leq j \leq J} e_{j} \log \left|Q_{j}(z)\right|,
$$

where the numbers $e_{j}$ and the polynomials $Q_{j}$ are as in Section 3 . We add to the previous set $S$ of 21 polynomials given in Table 2, the 10 polynomials of Table 4 . If $m$ is the minimum of $f(z)$ for $|z| \leq B$, by the same argument as in Section 3 we get

$$
s_{2}-e_{0} s_{1} \geq m d
$$

If we assume that $s_{1}$ has the value $\boldsymbol{\sigma}$, then $s_{2} \geq d m+e_{0} \boldsymbol{\sigma}$. We optimize the numbers $e_{0}, \ldots, e_{J}$ to get a maximal value for $d m+e_{0} \boldsymbol{\sigma}$. Therefore we get a lower bound for $s_{2}$ depending on the value of $\boldsymbol{\sigma}$. If we take $\boldsymbol{\sigma}$ close to its upper bound, then we get a bound for $s_{2}$ better than the one given in Section 3. If in (4.1) we replace $-e_{0} \operatorname{Re}(z)$ by $e_{0} \operatorname{Re}(z)$, we get the same lower bound for $s_{2}$ when $s_{1}$ has the value $-\boldsymbol{\sigma}$. We may also replace $\operatorname{Re}\left(z^{2}\right)$ by $-\operatorname{Re}\left(z^{2}\right)$ and get upper bounds for $s_{2}$. Then, replacing $B$ by $B^{k}$, we get bounds for $s_{2 k}$ when $s_{k}$ has values close to its bounds. We give a numerical example for $d=18$. For $k=6$ we have $-7 \leq s_{6} \leq 7$ and 
TABLE 4. List of new polynomials which are used in the auxiliary functions of Section 4a. Two different subsets of the polynomials $Q_{1}, \ldots, Q_{31}$ are used to get the upper bounds for $s_{2 k}$, respectively the lower bounds for $s_{2 k}$.

\begin{tabular}{lrrrrrrrr}
$Q_{j}$ & $d_{j}$ & \multicolumn{8}{c}{ Coefficients of $Q_{j}$} & \\
$Q_{22}$ & 1 & 1 & 1 & & & & & \\
$Q_{23}$ & 2 & 1 & 1 & 1 & & & & \\
$Q_{24}$ & 2 & 1 & 0 & -2 & & & & \\
$Q_{25}$ & 3 & 1 & -1 & -3 & 4 & & & \\
$Q_{26}$ & 3 & 1 & -1 & -4 & 5 & & & \\
$Q_{27}$ & 3 & 1 & -2 & -1 & 3 & & & \\
$Q_{28}$ & 3 & 1 & -3 & 1 & 3 & & & \\
$Q_{29}$ & 4 & 1 & 0 & 0 & 0 & 1 & & \\
$Q_{30}$ & 4 & 1 & 1 & 1 & 1 & 1 & & \\
$Q_{31}$ & 6 & 1 & 1 & 1 & 1 & 1 & 1 & 1
\end{tabular}

$-12 \leq s_{12} \leq 12$. Then we decrease the upper bounds of $s_{12}$ as follows:

$$
\begin{aligned}
\text { if }\left|s_{6}\right| & =7, \text { then } s_{12} \leq 3, \\
\text { if }\left|s_{6}\right| & =6, \text { then } s_{12} \leq 5, \\
\text { and if }\left|s_{6}\right| & =5, \text { then } s_{12} \leq 10
\end{aligned}
$$

For the lower bounds of $s_{12}$ we get

$$
\begin{aligned}
\text { if }\left|s_{6}\right| & \geq 6, \text { then } s_{12} \geq-9, \\
\text { if }\left|s_{6}\right| & =5, \text { then } s_{12} \geq-10, \\
\text { and if }\left|s_{6}\right| & =4, \text { then } s_{12} \geq-11 .
\end{aligned}
$$

Remark. The polynomial $P_{18}=X^{18}+X^{12}-1$, which is the minimal polynomial of the extremal $\boldsymbol{\alpha}$, does not satisfy these conditions since, for $P_{18}$, we have $s_{6}=-6$ and $s_{12}=6$. This is because we have used the polynomial $X^{3}-X^{2}+1$ in the auxiliary function and $-\boldsymbol{\alpha}^{6}$ is a root of this polynomial. If we do not use the polynomial $X^{3}-X^{2}+1$ in the auxiliary function, then for $\left|s_{6}\right|=6$ we have $s_{12} \leq 8$. So, for every $d$, we add to the set of polynomials obtained by our computations all the irreducible factors of degree $d$ of the polynomials $Q_{j}\left( \pm X^{k}\right)$.

When $d$ is large the results are more spectacular. For $d=28$ we have $\left|s_{42}\right| \leq 64$ and $\left|s_{84}\right| \leq 174$. If $\left|s_{42}\right|=64$, then $105 \leq s_{84} \leq 118$.

b. A study of the triples $\left(b_{d-2}, b_{d-1}, b_{d}\right)$.

Here we want to get good bounds for $\left|b_{d-2}\right|$ and $\left|b_{d-1}\right|$. Since $\left|b_{d}\right|=1$, we have $\left|b_{d-2}\right|=\left|\sum_{1 \leq i \leq d} 1 / \boldsymbol{\alpha}_{i}^{2}\right|$ and $\left|b_{d-1}\right|=\left|\sum_{1 \leq i \leq d} 1 / \boldsymbol{\alpha}_{i}\right|$. But we have $\left|1 / \boldsymbol{\alpha}_{i}\right| \leq B^{d-1}$, so this implies, when $d=28$ and $B=1.02245,\left|b_{d-1}\right| \leq d B^{d-1}<50.992$ and $\left|b_{d-2}\right| \leq d B^{2(d-1)}<92.87$. These bounds are unsatisfactory. Therefore, in order to obtain reasonable bounds for $b_{d-2}$ and $b_{d-1}$, we study $\boldsymbol{\sigma}_{k}=\sum_{1 \leq i \leq d} \boldsymbol{\alpha}_{i}^{k}+1 / \boldsymbol{\alpha}_{i}^{k}$ for $k=1,2$. The advantage is that the numbers $\boldsymbol{\alpha}_{i}^{k}+1 / \boldsymbol{\alpha}_{i}^{k}$ lie inside an ellipse which is "not too far" from the real axis. Moreover we will see that, in the worst case, all these numbers but one are inside an ellipse which is very close to the interval $[-2,2]$. Since the auxiliary functions $f(z)$ of the type (3.1) are more efficient when $z$ has a very small imaginary part, we will get good bounds for $\boldsymbol{\sigma}_{1}$ and $\boldsymbol{\sigma}_{2}$. For 
$d=28$ we get $\left|\sigma_{1}\right| \leq 8$ and $\left|\boldsymbol{\sigma}_{2}\right| \leq 13$. This gives, for a fixed pair $\left(s_{1}, s_{2}\right), 566$ triples.

We first need a lemma:

Lemma 2. Let $m$ be a positive real number, let $d \geq 2$ be an integer, and $h(x)$ a real continuous decreasing function defined on the interval $[0,(d-1) m]$. Let $h_{1}(x)$ and $h_{2}(x)$ be two linear functions such that:

For $0 \leq x \leq m, h(x)=h_{1}(x)$ and for $m \leq x \leq(d-1) m, h(x)=h_{2}(x)$.

Let $b_{1} \leq \ldots \leq b_{d} \leq m$ be $d$ real numbers such that

$$
\sum_{1 \leq i \leq d} b_{i}=0
$$

Define

$$
\omega=\sum_{1 \leq i \leq d} h\left(\left|b_{i}\right|\right)
$$

Then

$$
\omega \geq h((d-1) m)+(d-1) h(m) .
$$

Proof. If all the numbers $b_{i}$ vanish, then (4.1) is clearly true; otherwise there exist two integers $0 \leq l \leq k<d$ such that

$$
\begin{gathered}
-(d-1) m \leq b_{1} \leq \ldots \leq b_{l} \leq-m, \\
-m<b_{l+1} \leq \ldots \leq 0,
\end{gathered}
$$

and

$$
0<b_{k+1} \leq \ldots \leq b_{d} \leq m
$$

Now, if we put $a_{i}=\left|b_{i}\right|$ for all $i$, we have $a_{1}+\ldots+a_{k}=a_{k+1}+\ldots+a_{d}$ and $a_{1}+\ldots+a_{l} \geq l m$.

If $l=0$, then, since $h_{2}$ is linear, we have

$$
\omega \geq \sum_{1 \leq i \leq d} h_{1}(m)=\sum_{1 \leq i \leq d} h_{2}(m)=h_{2}((d-1) m)+(d-1) h_{2}(m) .
$$

If $l \geq 1$ we have

$$
\omega \geq \sum_{1 \leq i \leq l} h_{2}\left(a_{i}\right)+\sum_{l+1 \leq i \leq d} h_{1}(m) .
$$

Since $h_{2}$ is linear and

$$
l m \leq \sum_{1 \leq i \leq l} a_{i} \leq(d-k) m,
$$

the right-hand side of (4.2) is equal to

$$
h_{2}\left(\sum_{1 \leq i \leq l} a_{i}-(l-1) m\right)+(l-1) h_{2}(m)+(d-l) h_{1}(m) .
$$

Then, since $d-k-l+1 \leq d-1$, we have

$$
\omega \geq h_{2}((d-k-l+1) m)+(d-1) h_{2}(m) \geq h_{2}((d-1) m)+(d-1) h_{2}(m) .
$$

Let $P$ be a monic integer noncyclotomic polynomial of degree $d \geq 2$ such that $|P(0)|=1$. Let $\boldsymbol{\alpha}_{1}, \ldots, \boldsymbol{\alpha}_{d}$ be its roots in $\mathbb{C}$ and put $b_{i}=\frac{\log \left|\boldsymbol{\alpha}_{i}\right|}{\log B}$ with $b_{1} \leq \ldots \leq$ $b_{d} \leq 1$. Then there exists an integer $k \leq d-1$ such that $0<b_{k+1} \leq \ldots \leq b_{d} \leq 1$. 
Put $a_{i}=\left|b_{i}\right|$ for $1 \leq i \leq d$. Then $\boldsymbol{\alpha}_{i}+\frac{1}{\boldsymbol{\alpha}_{i}}$ is inside the ellipse $\mathcal{E}_{a_{i}}$ whose axes are $B^{a_{i}}+B^{-a_{i}}$ and $B^{a_{i}}-B^{-a_{i}}$.

Now let $f$ be an explicit auxiliary function of type (3.1). For $a \geq 0$ we define the function

$$
g(a)=\min _{z \in \mathcal{E}_{a}} f(z)
$$

Since $\boldsymbol{\alpha}_{i}+\frac{1}{\boldsymbol{\alpha}_{i}} \in \mathcal{E}_{a_{i}}$, we have

$$
\boldsymbol{\sigma}_{1} \geq \sum_{1 \leq i \leq d} g\left(a_{i}\right)
$$

Now we assume that we have a decreasing function $h$ which is continuous on the interval $[0, d-1]$, linear on both intervals $[0,1]$ and $[1, d-1]$ and such that, for all $a \in[0, d-1], h(a) \leq g(a)$. Then, By Lemma 2, we get from (4.4) that

$$
\sigma_{1} \geq(d-1) h(1)+h(d-1) .
$$

For a fixed $\varepsilon$ (say $\varepsilon=1 / 10$ ), we want the function $h$ to satisfy $h \leq g$ and to be such that

$$
(d-1) h(1)+h(d-1) \geq(d-1) g(1)+g(d-1)-\varepsilon .
$$

For $d=28$ and $B=1.02245$, we explain how to choose the function $h$. The polynomials $Q_{j}$ are the 11 polynomials $R_{j}$ given in Table 5 , and the coefficients $e_{j}$ are equal to

$0.16464679,0.49414790,0.61080711,0.04928365,0.03946975,0.21423746$,

$$
0.02691357,0.14143025,0.04972685,0.02489806,0.03963391 .
$$

We have $m_{1}=g(0)=-0.22094287 \ldots, m_{2}=g(1)=-0.22447363 \ldots, m_{3}=$ $g(d-1)=-2.83442434 \ldots$ and $(d-1) g(1)+g(d-1)=-8.89521261 \ldots$. We put $\varepsilon_{1}=\varepsilon_{2}=\frac{\varepsilon}{2(d-1)}$ and $\varepsilon_{3}=\frac{\varepsilon}{2}$.

The function $h_{1}$ is the line defined by $h_{1}(0)=m_{1}-\varepsilon_{1}$ and $h_{1}(1)=m_{2}-\varepsilon_{2}$. The function $h_{2}$ is defined by $h_{2}(1)=m_{2}-\varepsilon_{2}$ and $h_{2}(d-1)=m_{3}-\varepsilon_{3}$. We find a staircase function $s$ such that $h \leq s \leq g$. The sequence of points $0<a_{t}<\ldots<a_{1}=d-1$ where $s$ is not continuous is defined by induction as follows. We start at $a_{1}=d-1$ where $s\left(a_{1}\right)=g\left(a_{1}\right)$. Then $a_{2}<a_{1}$ is defined by $h_{2}\left(a_{2}\right)=g\left(a_{1}\right)$. Since $g$ and $h_{2}$ are decreasing we have, for any $a \in\left(a_{2}, a_{1}\right), h_{2}(a)<s(a)=g\left(a_{1}\right)<g(a)$. We continue until we obtain a point $a_{r-1}$ such that $m_{2}-\varepsilon_{2}<g\left(a_{r-1}\right)<m_{2}$. We take the next point $\left(a_{r}, g\left(a_{r-1}\right)\right)$ on the line $h_{1}$, and we continue the same process as before with $h_{1}$ instead of $h_{2}$, until $g\left(a_{t}\right) \geq m_{1}-\varepsilon_{1}$. The last stair is $\left(0, a_{t}\right)$ where $s$ has the value $g\left(a_{t}\right)$. In this case $t=31$ and $(d-1) h(1)+h(d-1) \geq-8.99521261 \ldots$ so $\sigma_{1} \geq-8$.

\section{The Final Computation}

The set $\mathcal{F}_{d}$ is very large when the degree $d$ increases. For $d=28$ it contains $1.6 \times 10^{12}$ polynomials. In this special case (and also for degree 26 ), for each of the 34 possible values of $\left(s_{1}, s_{2}\right)$ (restricted to $\left.s_{1} \leq 0\right)$, we first compute all possible triples $\left(b_{d-2}, b_{d-1}, b_{d}\right)$ and then compute the sets $\mathcal{F}_{d}$ and $\mathcal{F}_{3 d}$ relative to this pair $\left(s_{1}, s_{2}\right)$. If the set $\mathcal{F}_{3 d}$ is not empty, then we use the Schur-Cohn algorithm MAR] to compute the number of roots of $P$ inside a disc of radius equal to Matveev's bound (1.2). Since we always use a bound $B$ that will be subsequently proved to equal $\mathrm{m}(d)+\varepsilon$, we get few polynomials. For instance, in the case $d=26$ we 
TABLE 5. Polynomials $R_{j}$ which are used in the auxiliary functions of Section $4 \mathrm{~b} . d=\operatorname{deg} R_{j}$ and the coefficients of $R_{j}$ are written from degree $d$ to 0 .

\begin{tabular}{lrrrrrrrr}
$R_{j}$ & $d$ & & \multicolumn{7}{c}{ Coefficients of $R_{j}$} \\
$R_{1}$ & 1 & 1 & 0 & & & & \\
$R_{2}$ & 1 & 1 & 1 & & & & \\
$R_{3}$ & 1 & 1 & 2 & & & & \\
$R_{4}$ & 2 & 1 & 0 & -2 & & & \\
$R_{5}$ & 2 & 1 & 0 & -3 & & & \\
$R_{6}$ & 2 & 1 & 1 & -1 & & & \\
$R_{7}$ & 3 & 1 & 0 & -3 & 1 & & \\
$R_{8}$ & 3 & 1 & 1 & -2 & -1 & & \\
$R_{9}$ & 4 & 1 & 1 & -4 & -4 & 1 & \\
$R_{10}$ & 5 & 1 & -4 & -3 & -3 & 3 & 1 \\
$R_{11}$ & 5 & 1 & 1 & -5 & -5 & 4 & 3
\end{tabular}

get 11 polynomials. In the case $d=28$ we get 8 polynomials. For the very last computation we use Pari [PARI] to keep only the irreducible polynomials (which turn out to be always at most 1 ) and compute the roots of the polynomials $P$ to get $\nu$ and $\mathrm{m}(d)$.

In the case $d=3 k$ we get no polynomial at all, because the polynomial $X^{3}+$ $X^{2}-1$ is used inside one of the auxiliary functions, as was explained in Section 4a.

\section{REFERENCES}

[DB] D. Boyd, The Maximal Modulus of an Algebraic Integer, Math. Comp. 45 (1985), 243249. MR790657 (87c:11097)

[PB] P. Borwein, Computational Excursions in Analysis and Number Theory, CMS Books in Mathematics 10, Springer-Verlag, New York (2002). MR.1912495 (2003m:11045)

[D] A. Dubickas, On a conjecture of A. Schinzel and H. Zassenhaus, Acta Arith. 63 (1993), 15-20. MR.1201616 (94a:11161)

[FRSE] V. Flammang, G. Rhin and J.M. Sac-Épée, Integer transfinite diameter and polynomials of small Mahler measure, Math. Comp. 75 (2006), 1527-1540. MR2219043

[MAR] M. Marden, Geometry of polynomials, Amer. Math. Soc. Providence, Rhode Island (1966). MR0225972 (37:1562)

[MAT] E.M. Matveev, On the cardinality of algebraic integers, Math. Notes 49 (1991), 437-438. MR.1119233 (92g:11070)

[PARI $]$ C. Batut, K. Belabas, D. Bernardi, H. Cohen and M. Olivier, GP-Pari version 2.0.12, 1998.

[SZ] A. Schinzel and H. Zassenhaus, A refinement of two theorems of Kronecker, Michigan Math. J. 12 (1965), 81-85. MR0175882 (31:158)

[SM] C.J. Smyth, On the product of the conjugates outside the unit circle of an algebraic integer, Bull. London Math. Soc. 3 (1971), 169-175. MR0289451 (44:6641)

[V] P. Voutier, An effective lower bound for the height of algebraic numbers, Acta Arith. 74 (1996), 81-95. MR1367580 (96j:11098)

[WU1] Q. Wu, On the linear independence measure of logarithms of rational numbers, Math. Comp. 72 (2002), 901-911. MR.1954974 (2003m:11111)

[WU2] Q. Wu, The smallest Perron numbers (in preparation). 
UMR CNRS 7122, Département UfR Mim, Université de Metz, Ile du Saulcy, 57045 Metz Cedex 01, France

E-mail address: rhin@math.univ-metz.fr

Department of Mathematics, Southwest University of China, 2 Tiansheng Road Beibei, 400715 Chongqing, China

E-mail address: qiangwu@swu.edu.cn 